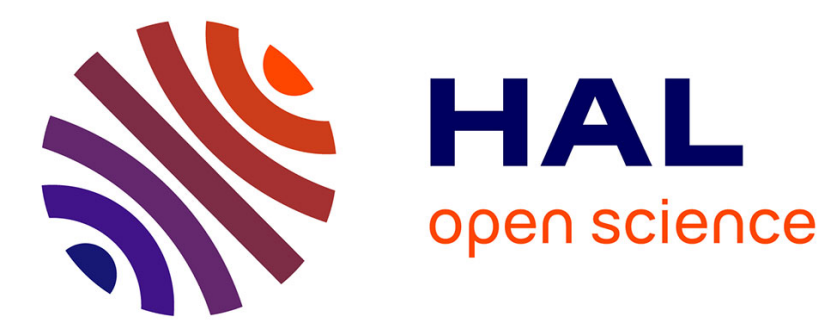

\title{
Caractérisation des défauts produits dans GaAs irradié aux protons par analyse des transitoires thermiques et optiques de capacité
}

\author{
G. Guillot, A. Nouailhat, G. Vincent, M. Baldy, A. Chantre
}

\section{To cite this version:}

G. Guillot, A. Nouailhat, G. Vincent, M. Baldy, A. Chantre. Caractérisation des défauts produits dans GaAs irradié aux protons par analyse des transitoires thermiques et optiques de capacité. Revue de Physique Appliquée, 1980, 15 (3), pp.679-686. 10.1051/rphysap:01980001503067900 . jpa-00244775

HAL Id: jpa-00244775

https://hal.science/jpa-00244775

Submitted on 1 Jan 1980

HAL is a multi-disciplinary open access archive for the deposit and dissemination of scientific research documents, whether they are published or not. The documents may come from teaching and research institutions in France or abroad, or from public or private research centers.
L'archive ouverte pluridisciplinaire HAL, est destinée au dépôt et à la diffusion de documents scientifiques de niveau recherche, publiés ou non, émanant des établissements d'enseignement et de recherche français ou étrangers, des laboratoires publics ou privés. 


\title{
Caractérisation des défauts produits dans GaAs irradié aux protons par analyse des transitoires thermiques et optiques de capacité (1) (2)
}

\author{
G. Guillot, A. Nouailhat, G. Vincent et M. Baldy \\ Laboratoire de Physique de la Matière $\left({ }^{3}\right)$, I.N.S.A. de Lyon, \\ 20, avenue A.-Einstein, 69621 Villeurbanne Cedex, France
}

\author{
et A. Chantre \\ C.N.E.T., BP 42, 38240 Meylan, France
}

\begin{abstract}
Résumé. - Nous avons analysé, par spectroscopie capacitive thermique (DLTS, conductance) les défauts créés à $300 \mathrm{~K}$ et à $77 \mathrm{~K}$ par irradiation de protons (énergie $90 \mathrm{keV}$, dose : quelque $10^{11} \mathrm{ions} / \mathrm{cm}^{2}$ ) dans des diodes Schottky $\mathrm{Au}-\mathrm{GaAs}$ de type $\mathrm{n}$. $\mathrm{A} 300 \mathrm{~K}$, les défauts produits sont les pièges à électrons $E_{2}, E_{3}, E_{4}, E_{5}$ trouvés après irradiation aux électrons. A dose d'implantation moyenne, un autre défaut $\left(E_{\mathrm{c}}-0,3 \mathrm{eV}, \sigma_{\mathrm{na}}=3 \times 10^{-14} \mathrm{~cm}^{2}\right)$ est mis en évidence. A $77 \mathrm{~K}$, un nouveau piège à électron $\left(E_{\mathrm{c}}-0,26 \mathrm{eV}, \sigma_{\mathrm{na}}=9 \times 10^{-13} \mathrm{~cm}^{2}\right)$ qui se recuit entre $200 \mathrm{~K}$ et $300 \mathrm{~K}$ est observé. Les défauts $\mathrm{E}_{2}$ et $\mathrm{E}_{3}$ sont aussi produits après une irradiation à basse température. Des résultats préliminaires concernant l'évolution de ces pièges lors des étapes de recuit entre $200 \mathrm{~K}$ et $300 \mathrm{~K}$ sont donnés. Nous avons également appliqué la nouvelle méthode de DLOS (Deep Level Optical Spectroscopy) à la mesure de la section efficace de capture optique $\sigma_{n}^{0}(h v)$ de $\mathrm{E}_{3}$, ce qui nous a permis de préciser l'effet de relaxation de réseau sur ce centre.
\end{abstract}

\begin{abstract}
Thermal capacitive spectroscopy (DLTS, conductance) have been performed on $\mathrm{n}$ type Gallium Arsenide Schottky barrier structures irradiated at $300 \mathrm{~K}$ and $77 \mathrm{~K}$ with one hundred keV protons at dose of some $10^{11} \mathrm{p}^{+} / \mathrm{cm}^{2}$. At $300 \mathrm{~K}$, the defects created are the electron traps $\mathrm{E}_{2}, \mathrm{E}_{3}, \mathrm{E}_{4}, \mathrm{E}_{5}$ found after electron irradiation. Another defect $\left(E_{\mathrm{c}}-0.3 \mathrm{eV}, \sigma_{\mathrm{na}}=3 \times 10^{-14} \mathrm{~cm}^{2}\right)$ is found at moderate dose. At $77 \mathrm{~K}$, a new electron trap $\left(E_{\mathrm{c}}-0.26 \mathrm{eV}, \sigma_{\mathrm{na}}=9 \times 10^{-13} \mathrm{~cm}^{2}\right)$ which anneals between $200 \mathrm{~K}$ and $300 \mathrm{~K}$ is detected. The $\mathrm{E}_{2}$ and $\mathrm{E}_{3}$ defects are also created after a low temperature irradiation. Preliminary results concerning the variations of these traps after the thermal annealing stages between $200 \mathrm{~K}$ and $300 \mathrm{~K}$ are described. We have also applied the new method of DLOS (Deep Level Optical Spectroscopy) to the determination of the optical capture cross section $\sigma_{n}^{0}(h v)$ of $\mathrm{E}_{3}$ which allowed us to precise the lattice relaxation effect of this centre.
\end{abstract}

1. Introduction. - GaAs est un composé semiconducteur largement utilisé aujourd'hui en électronique (transistor FET, diode laser, ...) et un travail considérable a été effectué sur la caractérisation des défauts profonds rencontrés dans ce matériau depuis l'apparition de la technique DLTS qui a permis une véritable spectroscopie thermique des défauts $[1,2,3]$. Actuellement, si cette phase de botanique est pratiquement terminée, très peu de ces défauts ont été identifiés quant à leur nature physique, de façon certaine [4].

( $\left.{ }^{1}\right)$ Conférence présentée au Congrès de la Société Française de Physique (Toulouse).

$\left.{ }^{2}\right)$ Travail financé par la Délégation Générale Scientifique et Technique (contrat $\mathrm{n}^{\mathrm{o}} 77.7 .1640$ ).

$\left({ }^{3}\right)$ Equipe de recherche associée au C.N.R.S.
L'irradiation apparaît alors comme un moyen d'étudier des défauts simples, introduits de façon contrôlée et sur lesquels on peut agir par plusieurs paramètres : température d'irradiation, énergie des particules incidentes, dose.

Seuls les niveaux produits par irradiation d'électrons à $300 \mathrm{~K}$ ont été étudiés par DLTS : cinq pièges à électrons $\left(E_{1}\right.$ à $\left.E_{5}\right)$ et un piège de trou $\left(\mathrm{H}_{1}\right)$ ont été détectés après irradiation de couches LPE [5]. Une quantité importante d'informations a été accumulée sur leur annihilation thermique [6], les variations de leur position en énergie dans le gap dans des composés comme $\mathrm{GaAl}_{x} \mathrm{As}_{1-x}$ [7], le couplage de ces défauts avec les phonons (accélération du taux de recuit par recombinaisons non radiatives sur le défaut) [8]. Un travail récent, entrepris par D. Pons $[9,10]$ a permis de déterminer les défauts créés par irradiation d'élec- 
trons à $300 \mathrm{~K}$ dans des couches VPE, leur annihilation thermique, leur taux d'introduction en fonction de l'énergie des électrons [11] et de préciser le couplage du piège $E_{3}$ avec le réseau à l'aide d'un modèle théorique interprétant les effets du champ électrique sur son taux d'émission $e_{\mathrm{n}}$ [12]. Néanmoins, jusqu'à présent, toutes les données spectroscopiques relatives aux défauts d'irradiation sont d'origine thermique : nous ne pouvons connaître que les énergies d'ionisation thermique $E_{\mathrm{n}}$ ou $E_{\mathrm{p}}$ respectives par rapport à la bande de conduction ou de valence, et les sections efficaces de capture des porteurs $\sigma_{\mathrm{n}}(T)$ et $\sigma_{\mathrm{p}}(T)$. Toutes les grandeurs optiques correspondantes $\left(E_{\mathrm{n}}^{0}, E_{\mathrm{p}}^{0}, \sigma_{\mathrm{n}}^{0}(h v)\right)$ et $\left.\sigma_{\mathrm{p}}^{0}(h v)\right)$ caractéristiques de ces défauts sont inconnues; or, elles contiennent des informations très importantes sur la structure de ces défauts (processus de relaxation, liaison avec les bandes). En outre, une importante question persiste à propos des centres produits à $300 \mathrm{~K}$ : est-ce que ce sont des défauts primaires qui apparaîtraient après irradiation à $77 \mathrm{~K}$ ou bien des défauts secondaires qui résulteraient de réactions entre défauts primaires lors des stades I et II d'annihilation (respectivement à $235 \mathrm{~K}$ et à $280 \mathrm{~K}$ ) qui ont été mis en évidence par Thommen sur des matériaux irradiés à $77 \mathrm{~K}$ [13] ?

Le but de ce travail est d'apporter des renseignements nouveaux sur les deux points précédents en analysant les défauts créés par implantation de protons à $300 \mathrm{~K}$ et pour la première fois à $77 \mathrm{~K}$, et en étudiant les propriétés optiques du niveau $\mathrm{E}_{3}$ par la méthode DLOS (Deep Level Optical Spectroscopy) récemment mise au point par $\mathrm{A}$. Chantre dans notre laboratoire [14, 15].

Nous avons choisi l'irradiation aux protons de basse énergie $(\sim 100 \mathrm{keV})$ pour créer des défauts car, d'une part, l'utilisation d'un petit accélérateur est bien adapté à toutes les expériences de caractérisation in situ à basse température et, d'autre part, les défauts peuvent être créés dans la région désertée de diodes Schottky (ou de jonction pn) : leurs différentes propriétés (détermination des signatures par DLTS, profils) peuvent être étudiées par les techniques capacitives.

2. Techniques expérimentales. - Deux plaquettes de GaAs obtenues par épitaxie en phase vapeur (VPE) avec des niveaux de dopage $n_{0}=2 \times 10^{16}$ et $5 \times 10^{16} / \mathrm{cm}^{3}$ (dopant soufre) ont été utilisées. Avant irradiation, les échantillons sont légèrement décapés (environ $1 \mu \mathrm{m}$ ) et une épaisseur de $300 \AA$ d'or est évaporée sur la surface. La surface des diodes Schottky est de $4,3 \times 10^{-2} \mathrm{~cm}^{2}$.

Les plaquettes sont désorientées d'environ $7^{\circ} \mathrm{par}$ rapport à l'axe du faisceau d'ions. Le vide régnant dans l'enceinte d'implantation est de $10^{-6}$ torr. Les doses sont mesurées avec une précision de 20 à $30 \%$.

Les irradiations de protons ont été effectuées à $300 \mathrm{~K}$ et à $77 \mathrm{~K}$ dans les conditions suivantes : énergie $E=90 \mathrm{keV}$, taux de dose $4 \mathrm{nA} / \mathrm{cm}^{2}$ ou $8 \mathrm{nA} / \mathrm{cm}^{2}$ (soit $2,5 \times 10^{10} \mathrm{p}^{+} / \mathrm{cm}^{2} . \mathrm{s}$ ou $5 \times 10^{10} \mathrm{p}^{+} / \mathrm{cm}^{2} . \mathrm{s}$ ), dose : entre $5 \times 10^{10}$ et $10^{12} \mathrm{p}^{+} / \mathrm{cm}^{2}$, température $=300 \mathrm{~K}$ ou $77 \mathrm{~K}$. Compte tenu du fait que la perte d'énergie de protons de $90 \mathrm{keV}$ dans l'épaisseur d'or est d'environ $10 \mathrm{keV}$ [16], la distribution des défauts créés sera caractérisée par une profondeur

$$
R_{\mathrm{p}}=0,7 \mu \mathrm{m} \quad \text { [17] } .
$$

Immédiatement après irradiation, les diodes Schottky sont analysées in situ à l'aide d'un dispositif expérimental conçu pour permettre la réalisation de toutes les méthodes photoélectriques de caractérisation de défauts. Un calculateur HP $9821 \mathrm{~A}$ pilote l'ensemble du dispositif expérimental. Cet ensemble permet d'effectuer :

\section{i) le profil de dopage $n(x)$ par $C(V)$;}

ii) l'analyse des transitoires thermiques de capacité et de courant. La capacité de la structure est mesurée par une détection synchrone PAR 129 A. Un Boxcar ATNE à deux portes d'échantillonnage permet l'enregistrement des courbes DLTS (gamme des taux d'émission thermique mesurables : $10^{-1} \mathrm{~s}^{-1}$ à $\left.300 \mathrm{~s}^{-1}\right)$. La gamme des taux d'émission peut être étendue jusqu'à $10^{6} \mathrm{~s}^{-1}$ soit par mesure des transitoires de courant à l'aide d'un préamplificateur PAR 113, soit par mesure de conductance [18] ;

iii) l'analyse des sections efficaces optiques $\sigma_{\mathrm{n}}^{0}(h v)$ et $\sigma_{\mathrm{p}}^{\mathrm{o}}(h v)$. A. Chantre et $\mathrm{D}$. Bois $[14,15]$ ont développé une procédure originale : la DLOS (Deep Level Optical Spectroscopy). La mesure de la dérivée initiale des transitoires de photocapacité d'une diode après illumination, pourvu que les conditions initiales aient été fixées (tous les centres pleins ou vides) permet la détermination des sections efficaces optiques $\sigma_{\mathrm{n}}^{0}(h v)$ et $\sigma_{\mathrm{p}}^{0}(h v)$ d'un défaut profond. Cette méthode réalise une véritable spectroscopie optique des défauts profonds au même titre que la DLTS réalise une spectroscopie thermique, avec une sensibilité équivalente. Dans le cas des défauts d'irradiation, la DLOS, grâce à sa sélectivité lorsque plusieurs niveaux sont en présence, apparaît comme la seule technique permettant une analyse optique par exemple pour les défauts créés par irradiation à $300 \mathrm{~K}$, il n'est pas possible d'isoler un niveau particulier car tous les défauts sont créés simultanément et se recuisent (sauf $E_{2}$ ) au cours du même stade d'annihilation [9].

3. Résultats expérimentaux. - 3.1 MesURE DE LA SECTION EFFICACE $\sigma_{\mathrm{n}}^{0}(h v)$ DU DÉFAUT $\mathrm{E}_{3}$. - Après avoir identifié le niveau $E_{3}$ sur un échantillon irradié aux électrons à $300 \mathrm{~K}$ (énergie $1 \mathrm{MeV}$, dose : $\left.2 \times 10^{16} \mathrm{e}^{-} / \mathrm{cm}^{2}\right)$ par son spectre DLTS, nous avons enregistré les spectres de DLOS électrique à $T=110 \mathrm{~K}$ et à $T=190 \mathrm{~K}$ de part et d'autre du pic DLTS de $\mathrm{E}_{3}$ (Fig. 1). Dans ce cas d'exploitation de la DLOS, la température est constante et la condition initiale sur la population des centres avant le transitoire de photocapacité est fixée en utilisant l'injection de 


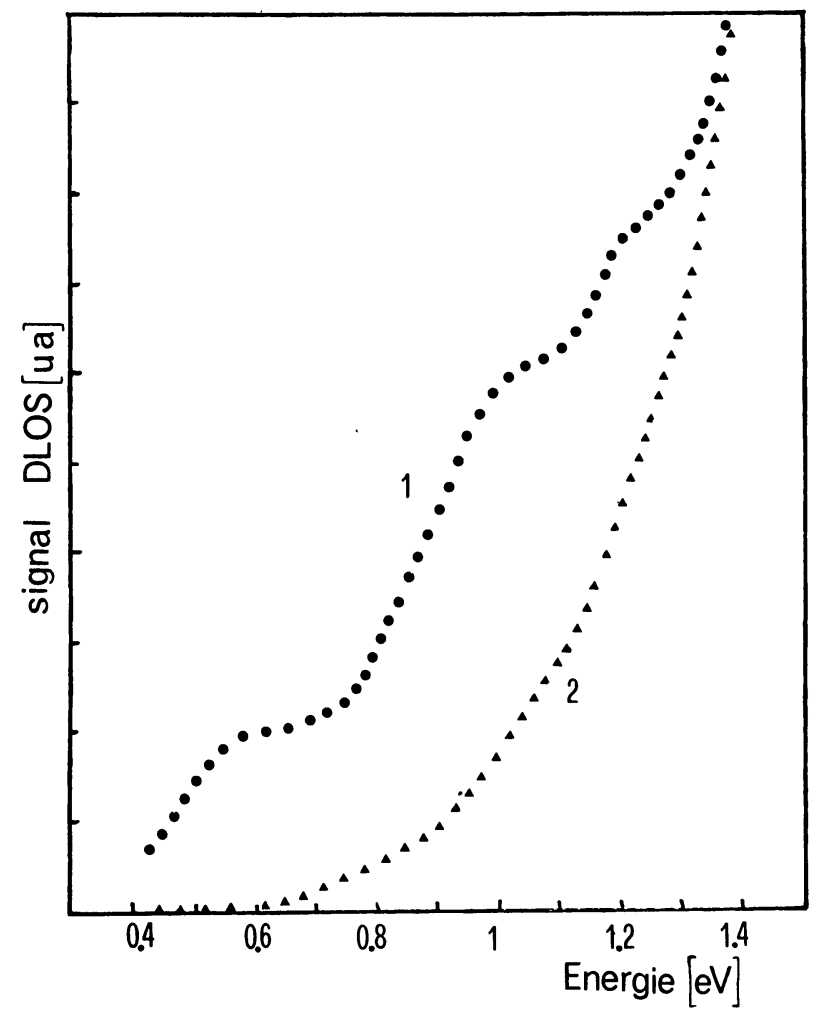

Fig. 1. - Spectres de DLOS électrique pour (1) à $T=110 \mathrm{~K}$, pour (2) à $T=190 \mathrm{~K}$ d'un échantillon GaAs (VPE) irradié aux électrons à $300 \mathrm{~K}\left(1 \mathrm{MeV}, 2 \times 10^{16} \mathrm{e}^{-} / \mathrm{cm}^{2}\right)$ (d'après [14]).

[Electrical DLOS spectra for (1) at $T=110 \mathrm{~K}$, for (2) at $T=190 \mathrm{~K}$ of a GaAs (VPE) electron irradiated at $300 \mathrm{~K}\left(1 \mathrm{MeV}, 2 \times 10^{16} \mathrm{e}^{-}\right.$ $\mathrm{cm}^{2}$ ) (from [14]).]

porteurs par des impulsions de tension. A $T=110 \mathrm{~K}$, trois bandes principales sont mises en évidence à $0,6 \mathrm{eV}, 1 \mathrm{eV}$ et $1,2 \mathrm{eV}$; le seuil d'une quatrième apparaît vers $1,3 \mathrm{eV}$. Ce spectre représente la somme des contributions de tous les niveaux non ionisés thermiquement à cette température $\left(\mathrm{E}_{3}, \mathrm{E}_{4}, \mathrm{E}_{5}\right)$ mais aussi des pièges à trou créés par l'irradiation [9]. A $T=190 \mathrm{~K}$, le niveau $\mathrm{E}_{3}$ ne contribue plus au spectre de DLOS électrique qui est alors dû aux pièges à électrons non ionisés thermiquement à cette température $\left(\mathrm{E}_{4}, \mathrm{E}_{5}\right)$ et aux mêmes pièges à trou qu'à $110 \mathrm{~K}$. Le spectre $\sigma_{\mathrm{n}}^{0}(h v)$, obtenu par différence des deux spectres de la figure 1 est donné sur la figure 2 et représente donc celui de la section efficace optique $\sigma_{\mathrm{n}}^{0}(h v)$ associé à $\mathrm{E}_{3}$.

3.2 NIVEAUX PROFONDS CRÉÉS PAR IRRADIATION DE PROTONS A $300 \mathrm{~K}$. - Il est bien connu que l'irradiation de GaAs par une dose suffisante de protons entraîne la formation en surface d'une couche compensée de haute résistivité [19] et ce phénomène est utilisé technologiquement pour créer des zones isolantes sur certains composants [20]. La figure 3 montre le profil de distribution $n(x)$ des porteurs libres obtenu après deux irradiations successives de $10^{11} \mathrm{p}^{+} / \mathrm{cm}^{2}$ et de $2 \times 10^{11} \mathrm{p}^{+} / \mathrm{cm}^{2}$ à $90 \mathrm{keV}$.

Le maximum du profil de concentration est situé

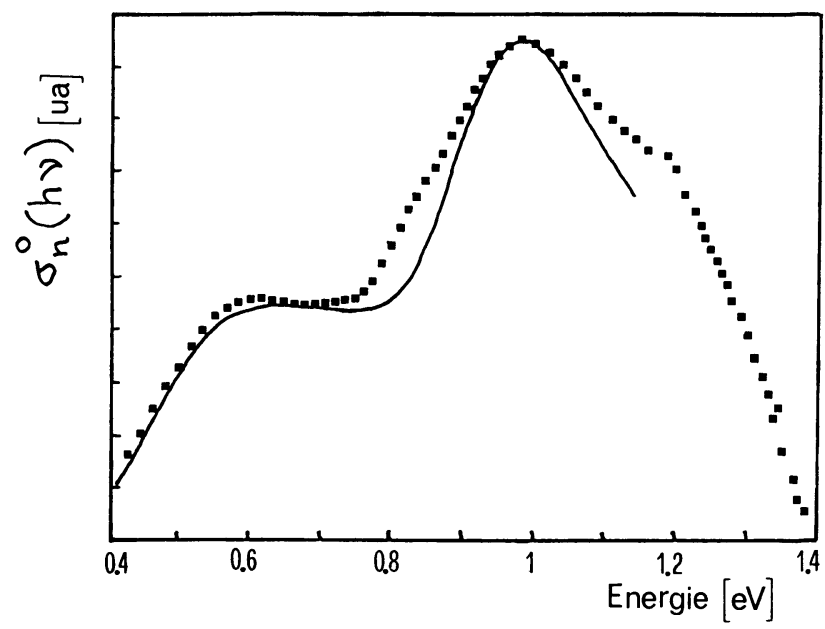

Fig. 2. - Section efficace optique $\sigma_{n}^{0}(h v)$ associée au niveau $\mathrm{E}_{3}$ d'après [14]. - : spectre obtenu par différence entre les spectres de DLOS électrique à $T=110 \mathrm{~K}$ et à $T=190 \mathrm{~K}$ de la figure 2; - : ajustement théorique obtenu pour les valeurs suivantes : $E_{\mathrm{n}}^{0}=0,44 \mathrm{eV}, d_{\mathrm{FC}} \simeq 0,2 \mathrm{eV}, \alpha^{-1}=8 \AA, P_{\mathrm{L}} / P_{\Gamma}=0 ; P_{\mathrm{X}} / P_{\Gamma}=0,15$; $P_{\Gamma}, P_{\mathrm{X}}, P_{\mathrm{L}}$ : poids de chaque extrémum $\Gamma, \mathrm{X}, \mathrm{L}$ (voir texte).

[Optical capture cross section associated to the $\mathrm{E}_{3}$ level from [14]. - : spectrum obtained by the difference between electrical DLOS spectra at $T=110 \mathrm{~K}$ and at $T=190 \mathrm{~K}$ of the figure $1 ;-$ theoretical fit with $E_{\mathrm{n}}^{0}=0.44 \mathrm{eV}, d_{\mathrm{FC}} \simeq 0.2 \mathrm{eV}, \alpha^{-1}=8 \AA$, $P_{\mathrm{L}} / P_{\Gamma}=0, P_{\mathrm{X}} / P_{\Gamma}=0.15 ; P_{\Gamma}, P_{\mathrm{X}}, P_{\mathrm{L}}:$ weight of each extremum $\Gamma$, $\mathrm{X}, \mathrm{L}$ (see text).]

environ à $x_{\mathrm{p}}=0,6 \mu \mathrm{m}$, ce qui correspond à la profondeur de pénétration de protons de $90 \mathrm{keV}$, compte tenu des pertes d'énergie dans l'or [16, 17]. Le matériau est compensé à $50 \%$ à $x_{\mathrm{p}}$ pour une dose de $10^{11} \mathrm{p}^{+} / \mathrm{cm}^{2}$ et à $80 \%$ pour une dose de $2 \times 10^{11} \mathrm{p}^{+} / \mathrm{cm}^{2}$. Par intégration sur le profil entier, nous obtenons un rapport $K$ (concentration intégrée des porteurs

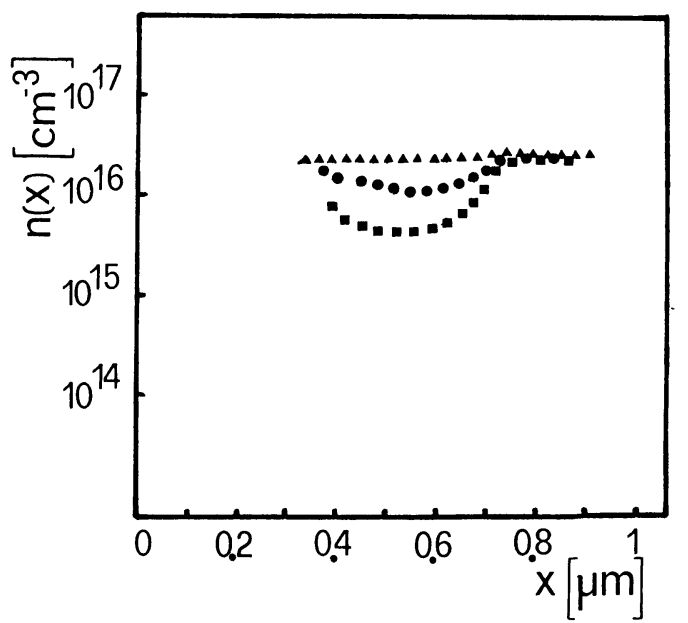

Fig. 3. - Concentration de porteurs libres $n(x)$ en fonction de la profondeur $x$ après irradiation de protons d'énergie $90 \mathrm{keV}$ à $300 \mathrm{~K}$ dans GaAs (VPE). $\Delta$ : matériau non irradié ; $:$ dose $10^{11} \mathrm{p}^{+} / \mathrm{cm}^{2}$; - : dose $2 \times 10^{11} \mathrm{p}^{+} / \mathrm{cm}^{2}$.

[Concentration of the free carriers $n(x)$ as a function of the depth $x$ after irradiation with protons of $90 \mathrm{keV}$ energy at $300 \mathrm{~K}$ in GaAs (VPE). $\Delta$ : unirradiated material; $\bullet$ : dose $10^{11} \mathrm{p}^{+} / \mathrm{cm}^{2}$; dose $2 \times 10^{11} \mathrm{p}^{+} / \mathrm{cm}^{2}$.] 
Tableau I.

$\begin{array}{ccccccc}n_{0} \text { du substrat } & \text { Ion } & \begin{array}{c}\text { Energie } \\ (\mathrm{keV})\end{array} & \begin{array}{c}\text { Dose } \\ \left(\mathrm{p}^{+} / \mathrm{cm}^{2}\right)\end{array} & \begin{array}{c}x_{\mathrm{p}} \\ (\mu \mathrm{m})\end{array} & K & \begin{array}{c}R_{\mathrm{p}} \\ (\mu \mathrm{m})\end{array} \\ \text { (ravail) } 2 \times 10^{16} & - & - & - & - & - & - \\ {[19] 1,3 \times 10^{16}} & \mathrm{H}^{+} & 90 & 10^{11} & 0,6 & 5 & 0,7 \\ & \mathrm{H}^{+} & 150 & 1,2 \times 10^{11} & 1,1 & 4 & 1,5\end{array}$

$x_{\mathrm{p}}$ : profondeur à laquelle le minimum de concentration de porteurs est mesurée,

$K$ : rapport de la concentration intégrée des porteurs compensés à la dose totale d'ions implantés,

$R_{\mathrm{p}}$ : profondeur projetée de pénétration des protons.

compensés/dose totale d'ions implantés) égal environ à $5 \mathrm{e}^{-} / \mathrm{p}^{+}$, ce qui signifie qu'en moyenne chaque proton incident de $90 \mathrm{keV}$ crée assez de défauts pour piéger 5 électrons. Dans le tableau I, nous avons regroupé, à titre de comparaison, les valeurs que nous avons trouvées et celles obtenues par Pruniaux [19]. La compensation maximum a lieu à une profondeur sensiblement égale à $R_{\mathrm{p}}$ et le phénomène physique responsable est le piégeage des porteurs libres par les défauts créés.

La figure 4 montre le spectre DLTS d'un échantillon irradié aux protons à $300 \mathrm{~K}$ qui met nettement en évidence 5 défauts dont les signatures $e_{\mathrm{n}}(T)$ sont données par les courbes de la figure 5. Trois des

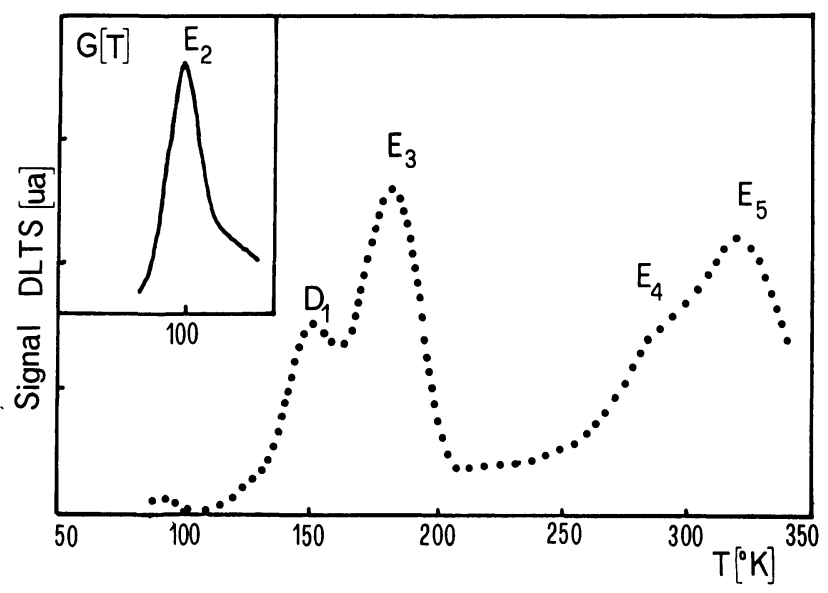

Fig. 4. - Spectre de DLTS d'une couche GaAs $\left(n_{0}=2 \times 10^{16} / \mathrm{cm}^{3}\right.$ VPE) irradiée aux protons à $300 \mathrm{~K}\left(90 \mathrm{keV}, 2 \times 10^{11} \mathrm{p}^{+} / \mathrm{cm}^{2}\right)$. Taux d'émission $e_{\mathrm{n}}=12.8 \mathrm{~s}^{-1}$. Encadré : pic de conductance associé au défaut $\mathrm{E}_{2}$ (fréquence : $10 \mathrm{kHz}$ ).

[DLTS spectrum of a GaAs VPE layer $\left(n_{0}=2 \times 10^{16} / \mathrm{cm}^{3}\right)$ irradiated with protons at $300 \mathrm{~K}\left(90 \mathrm{keV}, 2 \times 10^{11} \mathrm{p}^{+} / \mathrm{cm}^{2}\right)$. Window rate $: e_{\mathrm{n}}=12.8 \mathrm{~s}^{-1}$. Insert : conductance peak due to the $\mathrm{E}_{2}$ defect (frequency : $10 \mathrm{kHz}$ ).] pièges à électrons observés s'identifient aux niveaux $E_{3}, E_{4}, E_{5}$ observés par Lang [5] et Pons [9]. Le piège $\mathrm{E}_{2}$, plus superficiel, est observé en conductance (encadré de la figure 4).

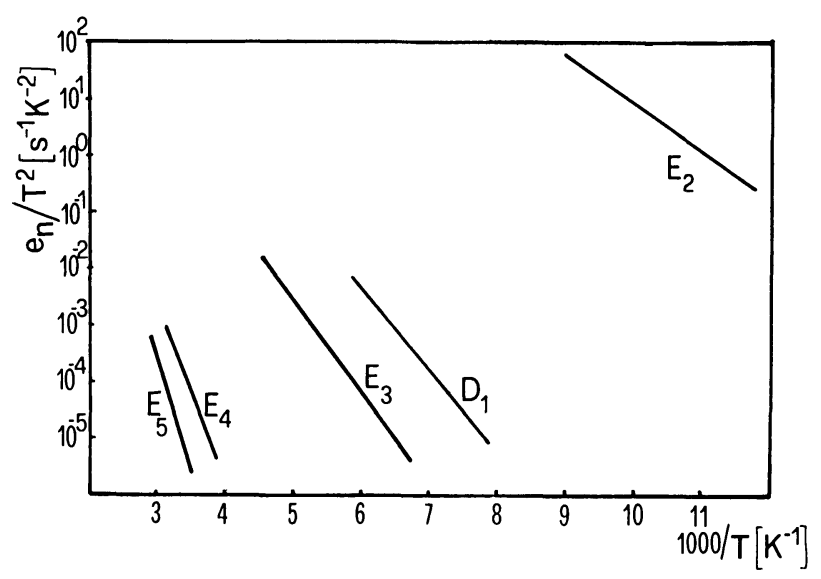

Fig. 5. - Signatures des niveaux crés par irradiation de protons (90 keV, dose : $2 \times 10^{11} \mathrm{p}^{+} / \mathrm{cm}^{2}$ ) dans GaAs VPE (mesures de conductance pour $\mathrm{E}_{2}$ ).

[Signatures of the defects created by proton irradiation $(90 \mathrm{keV}$, dose : $2 \times 10^{11} \mathrm{p}^{+} / \mathrm{cm}^{2}$ ) at $300 \mathrm{~K}$ in GaAs VPE (conductance measurements for $\left.E_{2}\right)$.]

Le tableau II résume les énergies d'activation thermique apparentes et les sections de capture apparentes que nous avons déduites des spectres DLTS pour les cinq niveaux.

Nous avons mis en évidence un nouveau défaut $D_{1}$ $\left(E_{\mathrm{c}}-0,3 \mathrm{eV}, \cdot \sigma_{\mathrm{na}}=3 \times 10^{-14} \mathrm{~cm}^{2}\right)$ dont la concentration est pratiquement égale à la moitié de celle de $E_{3}$ après une irradiation de $2 \times 10^{11} \mathrm{p}^{+} / \mathrm{cm}^{2}$.

3.3 NiVEAUX PROFONDS CRÉÉS PAR IRRADIATION DE PROTONS A $77 \mathrm{~K}$. - Il s'agit là de résultats tout

Tableau II. - Energies d'activation apparentes et sections de capture apparentes des pièges d'électrons produits par irradiation de protons à $300 \mathrm{~K}$.

[Apparent activation energies and capture cross sections of the electron traps produced by proton irradiation at $300 \mathrm{~K}$.]
$\mathrm{E}_{2}$
$\mathrm{D}_{1}$
$\mathrm{E}_{3}$
$\mathrm{E}_{4}$
$\mathrm{E}_{5}$
$E_{\text {na }}(\mathrm{eV})$
0,16
0,3
0,33
0,65
0,85
$\sigma_{\mathrm{na}}\left(\mathrm{cm}^{2}\right)$
$7 \times 10^{-12}$
$3 \times 10^{-14}$
$3 \times 10^{-15}$
$6,8 \times 10^{-14}$
$8 \times 10^{-12}$ 
à fait nouveaux puisque, à ce jour, aucune expérience spectroscopique n'a été réalisée sur les défauts d'irradiation créés à basse température dans GaAs.

Les pièges à électrons, produits à $77 \mathrm{~K}$, ont été étudiés en DLTS en se limitant à une zone de température comprise entre $77 \mathrm{~K}$ et $200 \mathrm{~K}$ à cause des stades de recuit I et II [13]. Par conséquent, seuls les états superficiels d'énergie comprise entre $\left(E_{\mathrm{c}}-0,15 \mathrm{eV}\right)$ et $\left(E_{\mathrm{c}}-0,4 \mathrm{eV}\right)$ peuvent être étudiés initialement.

3.3.1 Signature des défauts produits à $77 \mathrm{~K}$. La figure 6 montre un spectre DLTS typique mesuré dans ces conditions. Dans la fenêtre d'énergie étudiée, nous constatons la présence de trois défauts dont

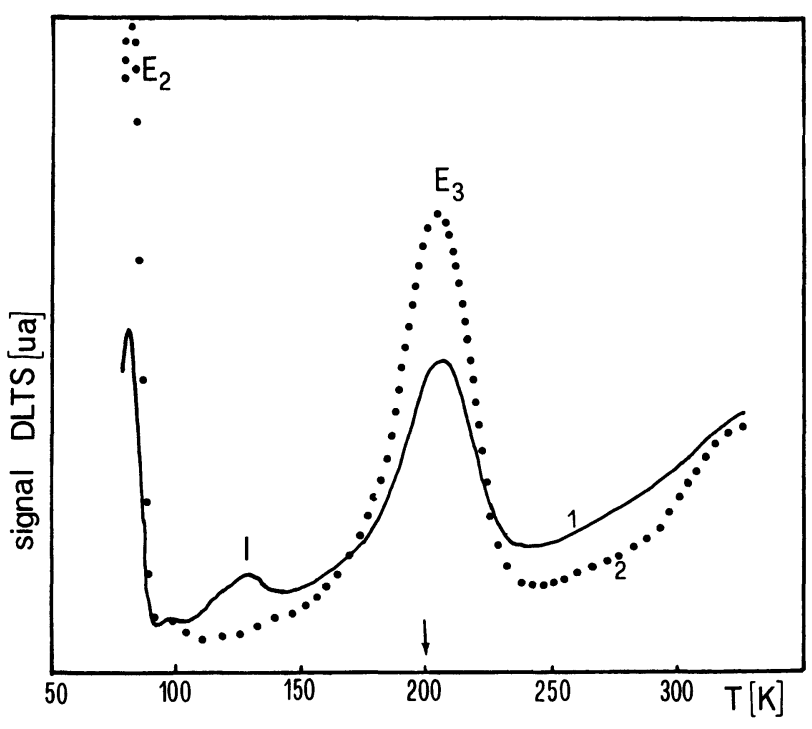

Fig. 6. - Spectres de DLTS d'une couche GaAs $\left(n_{0}=2 \times 10^{16} / \mathrm{cm}^{3}\right.$, VPE) irradiée aux protons à $77 \mathrm{~K}\left(95 \mathrm{keV}, 8 \times 10^{10} \mathrm{p}^{+} / \mathrm{cm}^{2}\right)$. Taux d'émission $e_{\mathrm{n}}=274,6 \mathrm{~s}^{-1}$. - : spectre DLTS après irradiation à $77 \mathrm{~K}$; : spectre DLTS après un recuit de $15 \mathrm{~min}$ à $320 \mathrm{~K}$; $\downarrow$ : température limite des cycles thermiques pour l'étude DLTS des défauts créés à $77 \mathrm{~K}$ (voir texte).

[DLTS spectra of a GaAs layer $\left(n_{0}=2 \times 10^{16} / \mathrm{cm}^{3}, \mathrm{VPE}\right)$ proton irradiated at $77 \mathrm{~K}\left(95 \mathrm{keV}, 8 \times 10^{10} \mathrm{p}^{+} / \mathrm{cm}^{2}\right)$. Window rate : $274.6 \mathrm{~s}^{-1}$. — : DLTS spectrum after irradiation at $77 \mathrm{~K}$; DLTS spectrum after annealing $(15 \mathrm{~min}$. at $320 \mathrm{~K}) ; \downarrow$ : upper temperature of the thermal cycles for the study of irradiation defects formed at $77 \mathrm{~K}$ (see text).]

les signatures sont rassemblées dans le tableau III, ainsi que sous forme graphique (Fig. 7). Deux de ces pièges sont les niveaux $E_{2}$ et $E_{3}$ créés par irradiation à $300 \mathrm{~K}$. Le troisième défaut noté $\mathrm{I}$ est reporté pour la première fois et apparaît spécifique d'une irradiation à l'azote liquide.

3.3.2 Influence d'un recuit à $300 \mathrm{~K}$. - Un recuit à température ambiante produit trois effets majeurs :

i) la diminution de la compensation;

ii) l'annihilation complète du défaut I (Fig. 6);

iii) une augmentation de $(\Delta C / C)_{i} i=2,3$ correspondant aux pics DLTS des défauts $\mathrm{E}_{2}$ et $\mathrm{E}_{3}$ (Fig. 6).

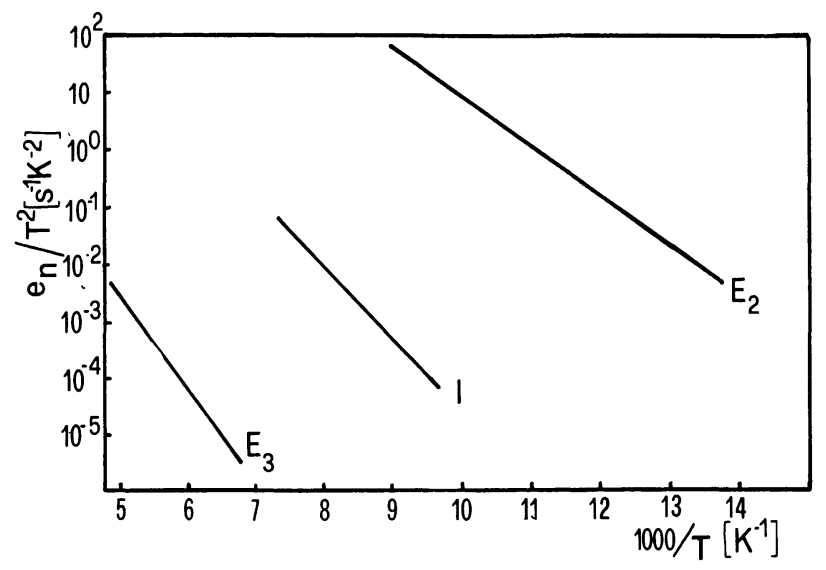

Fig. 7. - Signatures des défauts créés à $77 \mathrm{~K}$ (domaine de balayage en température $77 \mathrm{~K}-200 \mathrm{~K} ; \mathrm{E}_{2}$ étudié par DLTS et conductance).

[Signatures of the defects created at $77 \mathrm{~K}$ (temperature sweeping range $77 \mathrm{~K}-200 \mathrm{~K} ; \mathrm{E}_{2}$ studied by DLTS and conductance).]

Tableau III. - Energies d'activation apparentes et sections de capture apparentes des pièges d'électrons produits par irradiation de protons à $77 \mathrm{~K}$.

[Apparent activation energies and capture cross sections of the electron traps produced by proton irradiation at $77 \mathrm{~K}$.]

$$
\begin{array}{lccc} 
& \mathrm{E}_{2} & I & \mathrm{E}_{3} \\
E_{\text {na }}(\mathrm{eV}) & - & - & - \\
\sigma_{\mathrm{na}}\left(\mathrm{cm}^{2}\right) & 7 \times 16 & 0,26 & 0,35 \\
& 7 \times 10^{-12} & 9 \times 10^{-13} & 1,5 \times 10^{-14}
\end{array}
$$

Le tableau IV résume les valeurs obtenues pour les variations de $(\Delta C / C)_{i}$, la largeur de la zone désertée à $-2 \mathrm{~V}$ (tension inverse appliquée à la diode) avant et après recuit.

Les pics DLTS, associés aux niveaux $\mathrm{E}_{4}$ et $\mathrm{E}_{5}$, n'apparaissent pas clairement après irradiation à $77 \mathrm{~K}$ et recuit à température ambiante.

Remarque. - La structure des pics DLTS liés à $\mathrm{E}_{4}$ et à $\mathrm{E}_{5}$ est apparue cependant nettement après un recuit prolongé à $300 \mathrm{~K}$ (Fig. 8) dans deux cas sur cinq sans qu'on puisse donner, actuellement, d'explication à ce phénomène.

Tableau IV. - Valeurs obtenues pour $(\Delta C / C)_{i}$, la largeur de la zone désertée l $\grave{a}-2 \mathrm{~V}$ après irradiation à $77 \mathrm{~K}$ et après recuit à $320 \mathrm{~K}$.

[Values of $(\Delta C / C)_{i}$, of the depletion depth $l$ at $-2 \mathrm{~V}$ after an irradiation at $77 \mathrm{~K}$ and a thermal anneal at $320 \mathrm{~K}$.]

$\begin{array}{lcc}l(-2 \mathrm{~V})(\mu \mathrm{m}) & \overline{0,95} & \overline{0,82} \\ (\Delta C / C)_{2} & 10^{-2} & 1,75 \times 10^{-2} \\ (\Delta C / C)_{3} & 9 \times 10^{-3} & 1,25 \times 10^{-2} \\ x_{\mathrm{p}}(\mu \mathrm{m}) & 0,65 & 0,65\end{array}$




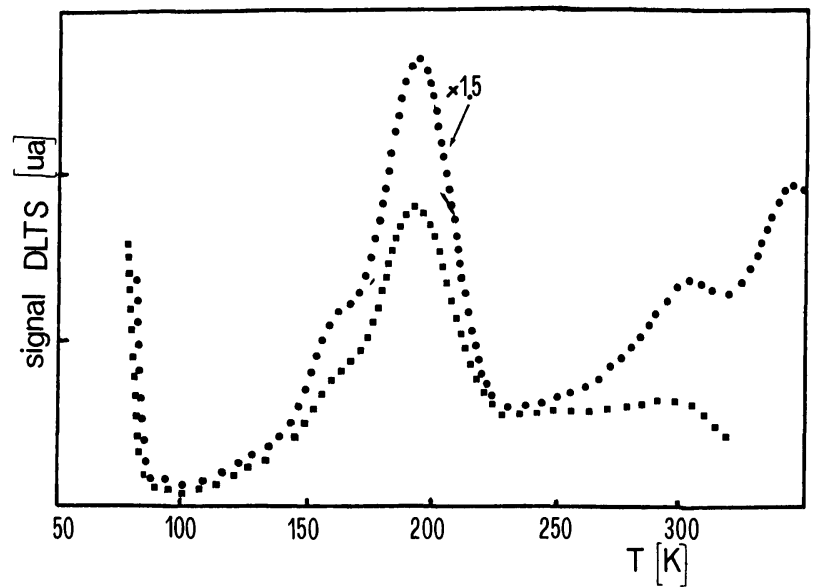

Fig. 8. - Spectres de DLTS d'une couche GaAs $\left(n_{0}=2 \times 10^{16} / \mathrm{cm}^{3}\right.$, VPE) irradiée aux protons à $77 \mathrm{~K}\left(90 \mathrm{keV}, 6 \times 10^{10} \mathrm{p}^{+} / \mathrm{cm}^{2}\right)$. Taux d'émission : $64 \mathrm{~s}^{-1}$ ( $\square$ : après recuit de $5 \min$ à $50^{\circ} \mathrm{C}$; après un recuit de $72 \mathrm{~h}$ à $300 \mathrm{~K}$ ).

[DLTS spectra of a GaAs layer $\left(n_{0}=2 \times 10^{16} / \mathrm{cm}^{3}\right.$, VPE) proton irradiated at $77 \mathrm{~K}\left(90 \mathrm{keV}, 6 \times 10^{10} \mathrm{p}^{+} / \mathrm{cm}^{2}\right)$. Window rate : $64 \mathrm{~s}^{-1}$ ( : : after a thermal anneal of $5 \mathrm{~min}$. at $50{ }^{\circ} \mathrm{C}: \bullet$ : after a thermal anneal of $72 \mathrm{~h}$ at $300 \mathrm{~K})$.]

4. Discussion. - 4.1 ANALYSE DE LA SECTION EFFICACE OPTIQUe $\sigma_{\mathrm{n}}^{0}(h v)$ DE $\mathrm{E}_{3}$. - A. Chantre et D. Bois $[14,15]$ ont élaboré un modèle théorique permettant d'exploiter toute l'information physique contenue dans les spectres de DLOS (détermination de l'énergie d'ionisation optique $E_{\mathrm{n}}^{0}\left(\right.$ ou $\left.E_{\mathrm{p}}^{0}\right)$, contribution des différentes bandes, ...). Pour les détails $\mathrm{du}$ modèle, nous renvoyons aux travaux $[14,15]$ et nous nous limiterons, ici, à donner les résultats de l'analyse de la section efficace optique $\sigma_{\mathrm{n}}^{0}(h v)$ de $\mathrm{E}_{3}$.

Le spectre $\sigma_{\mathrm{n}}^{0}(h v)$ théorique, ainsi que le spectre expérimental sont donnés sur la figure 2 . Le fittage a été réalisé avec les valeurs suivantes des paramètres :

- énergie d'ionisation optique : $E_{\mathrm{n}}^{0}=0,44 \mathrm{eV}$,

- paramètre de Franck Condon :

$$
d_{\mathrm{FC}}=E_{\mathrm{n}}^{0}-E_{\mathrm{n}} \simeq 0,2 \mathrm{eV},
$$

- étendue de la fonction d'onde de l'électron sur le piège : $\alpha^{-1}=8 \AA$,

- poids relatifs des transitions vers $\mathrm{L}$ et $\mathrm{X}$ par rapport à celles vers $\Gamma: P_{\mathrm{L}} / P_{\Gamma}=0 ; P_{\mathrm{X}} / P_{\Gamma}=0,15$.

On observe un assez bon accord entre les deux spectres.

La figure 9 indique, en diagramme de configuration, les énergies thermiques et optiques caractéristiques de l'émission de l'électron du niveau $\mathrm{E}_{3}$ vers la bande de conduction déterminées en DLTS et DLOS. Malgré une incertitude sur l'énergie thermique à cause des effets de champ électrique [12], il apparaît clairement que $\mathrm{E}_{3}$ correspond à un cas de relaxation classique dans les composés III-V [15]. Ce résultat est en contradiction avec l'hypothèse de Lang qui a suggéré récemment que ce défaut est le siège d'une forte relaxation de réseau près de la bande de valence, après capture d'un électron, ce qui permet d'expliquer

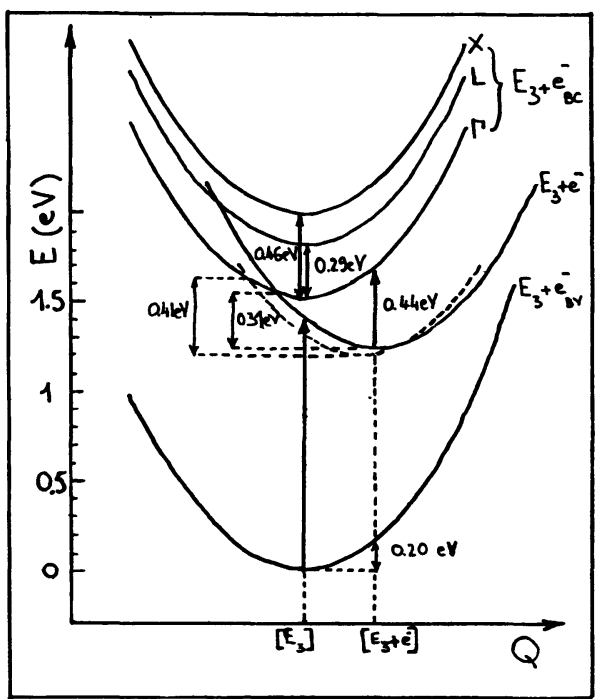

Fig. 9. - Diagramme de configuration pour le niveau $\mathrm{E}_{3}$ : énergie électronique et élastique en fonction de la coordonnée de configuration $Q$. (d'après [14]).

[Configuration coordinate diagram for the $\mathrm{E}_{3}$ defect : electronic and elastic energy as a function of the configuration coordinate $Q$ (from [14]).]

sa forte section efficace de capture de trous $\left(\sigma_{\mathrm{p}} \simeq 5 \times 10^{-15} \mathrm{~cm}^{2}\right)$ pour un niveau apparemment aussi proche de la bande de conduction [8].

La figure 9 prévoit une hauteur de barrière de 20 à $100 \mathrm{meV}$ pour la capture d'électrons par émission multiphonon (MPE) sur le niveau $\mathrm{E}_{3}$. L'énergie d'activation thermique de la section efficace de capture $\sigma_{\mathrm{n}}$ mesurée par Lang, $100 \mathrm{meV}$ [8] semble d'ailleurs montrer que, dans ce cas, le mécanisme MPE est effectivement mis en jeu.

4.2 Défauts CRÉÉS PAR IRRADIATION DE PROTONS A $300 \mathrm{~K}$. - Les irradiations d'électrons ou de protons à $300 \mathrm{~K}$ (en se limitant aux doses faibles $\left.\left(\leqslant 10^{11} \mathrm{p}^{+} / \mathrm{cm}^{2}\right)\right)$ conduisent à des défauts identiques avec, toutefois, deux différences notables que nous avons mises en évidence :

i) la comparaison des rapports des concentrations des pièges $E_{3}$ par rapport à celle de $E_{4}$ et $E_{5}$ dans les échantillons irradiés aux électrons et aux protons, montre que le rendement de formation des défauts $E_{4}$ et $E_{5}$ par rapport au défaut $E_{3}$ est plus élevé d'un facteur 5 pour des irradiations aux protons;

ii) le défaut $D_{1}$ qui n'apparaît pas lors d'irradiations de couches LPE et VPE aux électrons, est créé par implantation de protons aux doses moyennes. Il pourrait donc s'agir d'un nouveau défaut spécifique des implantations de protons car on s'attend à ce que les particules plus lourdes que les électrons (proton, neutron, ion) créent des défauts ayant une structure plus complexe. Nous devons noter que ce défaut $\mathrm{D}_{1}$ a une signature identique au piège d'électron EL7 $\left(E_{\mathrm{c}}-0,3 \mathrm{eV}, \sigma_{\mathrm{na}}=10^{-14} \mathrm{~cm}^{2}\right)$ spécifique des couches de GaAs obtenues par MBE [3]. 
Le point i) pourrait s'expliquer par le fait que les défauts $\mathrm{E}_{4}$ et $\mathrm{E}_{5}$ sont des défauts plus complexes que $E_{2}$ et $E_{3}$, hypothèse qui semble, néanmoins, contredite par plusieurs résultats : - la concentration de ces défauts croît linéairement avec la dose d'électrons indépendamment de la concentration en impuretés [6] - ils possèdent le même seuil de création en énergie que les défauts $\mathrm{E}_{2}$ et $\mathrm{E}_{3}$ [11]. Le comportement sous recuit de $\mathrm{E}_{5}$ est identique à celui de $\mathrm{E}_{3}$ qui est certainement un défaut simple [9]. Toutefois, le fait que les niveaux $\mathrm{E}_{4}$ et $\mathrm{E}_{5}$ ne semblent pas être directement produits lors d'une irradiation à $77 \mathrm{~K}$ tendrait à prouver que ces défauts ne sont pas des défauts simples.

4.3 DÉFAUTS CRÉÉS PAR IRRADIATION DE PROTONS A $77 \mathrm{~K}$. - L'analyse DLTS des défauts produits à $77 \mathrm{~K}$ montre que les pièges $E_{2}$ et $E_{3}$ sont créés lors d'une irradiation à basse température et ne résultent donc pas de réactions secondaires ayant lieu lors des stades de recuit I et II. Ce résultat important est une preuve supplémentaire du fait que $E_{2}$ et $E_{3}$ sont certainement des défauts simples, et est en accord avec les hypothèses de D. Pons et A. Zylberstejn suivant lesquelles le défaut $E_{2}$ serait une lacune [9] et $\mathrm{E}_{3}$ le défaut d'antisite $\mathrm{As}_{\mathrm{Ga}}$ [21].

D'après les résultats de Thommen [13] et de D. Pons [11] sur les variations du taux d'introduction des défauts en fonction de l'énergie, il est probable que la dilacune est créée lors d'une irradiation à $77 \mathrm{~K}$ : il pourrait s'agir du nouveau défaut I. L'étude du taux d'introduction de ce défaut, en fonction de l'énergie des électrons incidents, pourrait apporter une conclusion définitive sur ce point.

Nous avons cherché à préciser si les augmentations de $(\Delta C / C)_{2,3}$ lors du recuit (Tableau IV) sont significatives d'une croissance de la concentration des défauts $E_{2}$ et $E_{3}$ ou traduisent simplement le fait que par suite de la diminution de la compensation après recuit, la zone désertée $l$ varie $\left(l \rightarrow l_{R}\right)$. En effet, si on suppose, d'une part, que la distribution de chaque défaut $i$ peut être représentée par une fonction $f(x)$, identique pour tous les défauts, que multiplie la valeur $E_{i}$ de la concentration au maximum, et que, d'autre part, seul $E_{i}$ est susceptible de varier au cours du recuit $\left(E_{i} \rightarrow E_{i \mathrm{R}}\right)$, alors le rapport $\eta_{i}$ des valeurs de $(\Delta C / C)_{i}$ relatives au défaut $i$ après et avant recuit doit être au plus égal à :

$$
\eta_{i}=\frac{E_{i \mathrm{R}}}{E_{i}}\left(\frac{l}{l_{\mathrm{R}}}\right)^{2} .
$$

$\eta_{i}$ est la valeur que l'on doit avoir strictement si tous les défauts $i$ créés sont à l'intérieur de la zone dont la profondeur est déterminée par le point de croisement entre le niveau de Fermi et $\left(E_{\mathrm{T}}\right)_{i}$ niveau d'énergie de ces centres.

Suivant (1) et les valeurs du tableau IV, pour $\mathrm{E}_{2}$ nous observons une augmentation anormale du rapport $\eta_{2}\left(\eta_{2}=1,75\right)$ par rapport à la variation de $l$ : il semblerait, par conséquent, que la concentration de $\mathrm{E}_{2}$ augmente environ de $30 \%$ lors du recuit alors que celle de $E_{3}$ reste constante. En effet, pour $E_{3}$, $\eta_{3}$ est sensiblement égal à $\left(l / l_{\mathrm{R}}\right)^{2}$.

Cette conclusion semble, néanmoins, spéculative et demande à être confirmée par des études systématiques en fonction de divers paramètres (tension de polarisation, énergie des protons, dose, ...). Si l'augmentation de $E_{2}$ a lieu effectivement lors d'un recuit à $300 \mathrm{~K}$, celle-ci pourrait s'interpréter par le recuit de la dilacune qui, lors des stades I et II d'annihilation se recombinerait avec un défaut intersticiel voisin $\left(\mathrm{As}_{\mathrm{i}}\right.$ ou $\left.\mathrm{Ga}_{\mathrm{i}}\right)$, ce qui donnerait :

- soit une lacune simple $\left(\mathrm{V}_{\mathrm{Ga}}\right.$ ou $\left.\mathrm{V}_{\mathrm{As}}\right)$, ce qui augmenterait la concentration de $\mathrm{E}_{2}$,

- soit une association d'une lacune simple et d'un défaut d'antisite $\left(\mathrm{V}_{\mathrm{As}}+\mathrm{As}_{\mathrm{Ga}}\right.$ ou $\left.\mathrm{V}_{\mathrm{Ga}}+\mathrm{Ga}_{\mathrm{As}}\right)$.

Le fait que la concentration du piège I est comparable avec l'augmentation de $E_{2}$ est en faveur de cette hypothèse.

5. Conclusion. - En utilisant les mesures spectroscopiques (DLTS et DLOS) sur les transitoires thermiques et optiques de capacité, nous avons étudié les pièges à électrons créés dans GaAs (VPE) par une implantation de protons à $300 \mathrm{~K}$ et à $77 \mathrm{~K}$.

Nous avons ainsi pu montrer que, à dose faible, les défauts produits à $300 \mathrm{~K}$ sont rigoureusement identiques à ceux créés par une irradiation d'électrons. Un nouveau défaut a été mis nettement en évidence après une implantation à dose moyenne (entre $10^{11}$ et $10^{12} \mathrm{p}^{+} / \mathrm{cm}^{2}$ ).

Pour la première fois, les niveaux introduits par une irradiation à basse température ont été caractérisés : les défauts $E_{2}$ et $E_{3}$ sont créés ce qui confirme que ces pièges sont des défauts simples. Un nouveau défaut se recuisant entre $200 \mathrm{~K}$ et $300 \mathrm{~K}$ a été identifié : les variations du taux d'introduction de ce défaut avec l'énergie des électrons dans GaAs de type $n$ irradié à la température de l'azote liquide pourraient confirmer, de façon directe, l'identification de ce défaut avec la dilacune.

D'une part, la comparaison des taux d'introduction du niveau $E_{3}$ avec ceux des niveaux $E_{4}$ et $E_{5}$ après irradiation d'électrons et de protons et, d'autre part, le fait que les niveaux $E_{4}$ et $E_{5}$ ne semblent pas créés directement à $77 \mathrm{~K}$ indiquerait qu'il s'agit de défauts plus complexes qu'il n'a été supposé jusqu'à présent.

L'analyse de la section efficace optique de $E_{3}$ mesurée par DLOS a permis de préciser les effets de relaxation de réseau sur ce niveau et de le classer dans les défauts à relaxation de réseau normale.

Remerciements. - Nous tenons à remercier ici M. G. M. Martin du L.E.P. pour nous avoir fourni les échantillons de GaAs (VPE), M. D. Pons et M. J. C. Bourgoin pour l'irradiation électronique, ainsi que la D.G.R.S.T. pour son soutien financier (contrat $\mathrm{n}^{\circ}$ 77.7.1640). 


\section{Bibliographie}

[1] Lang, D. V., Logan, R. A., J. Electron. Mater. 4 (1975) 1053.

[2] Mitonneau, A., Martin, G. M., Mircea, A., Electron. Lett. 13 (1977) 666.

[3] Martin, G. M., Mitonneau, A., Mircea, A., Electron. Lett. 13 (1977) 191.

[4] Mircea, A., Bois, D., Int. Conf. on Rad. Effects in Semicond. Nice (1978).

[5] Lang, D. V., Kimmerling, L. C., Lattice Defects in Semicond. 1974, Inst. Phys. Conf. Ser. 23 (1975), p. 581.

[6] Lang, D. V., Rad. Effect in Semicond. 1976, Inst. Phys. Conf. Ser. 31 (1977), p. 70.

[7] Lang, D. V., Logan, R. A., Kimmerling, L. C., Phys. Rev. B 15 (1977) 10, 4874.

[8] Lang, D. V., Henry, C. H., Phys. Rev. Lett. 35 (1975) 22, 1525.

[9] Pons, D., Thèse Docteur-Ingénieur, Université de Paris (1979).

[10] Pons, D., Mircea, A., Mitonneau, A., Martin, G. M., Int. Conf. on Rad. Effects in Semicond. Nice (1978).
[11] Pons, D., Mooney, P. M., Bourgoin, J. C., à paraitre dans J. Appl. Phys.

[12] Makram, S. Ebeid, Pons, D., soumis à Phys. Rev.

[13] Thommen, K., Radiat. Eff. 2 (1970) 201.

[14] Chantre, A., Thèse Docteur-Ingénieur, Université de Grenoble (1979).

[15] Bois, D., Chantre, A., Conf. SFP Toulouse (1979).

[16] The stopping and ranges of ions in matter 3 ed. J. F. Ziegler (Pergamon Press) 1977.

[17] Matsumura, H., Nagatomo, M., Furukawa, S., Stephens, K. G., Radiat. Eff. 13 (1977) 121.

[18] Vincent, G., Bois, D., Pinard, P., J. Appl. Phys. 46 (1975) 5173.

[19] Pruniaux, B. R., North, J. C., Miller, G. L., 2nd Int. Conf. on Ion Implantation in Semiconductors (Springer Verlag), 1971, 212.

[20] Foyt, A. G., Lindley, W. T., Wolfe, C. M., Donnelly, J. P., Solid State Electron. 12 (1969) 209.

[21] Zylbersztejn, A., Int. Conf. on Deep Levels in Semiconductors, Sainte Maxime (1979). 